\title{
Energy Saving Strategies for Mobile Devices using Wake-up Signals
}

\author{
Gian Paolo Perrucci \\ Aalborg University \\ Dept. of Electronic Systems \\ Aalborg - Denmark \\ gpp@es.aau.dk \\ Giovanni Sasso \\ Aalborg University \\ Dept. of Electronic Systems \\ Aalborg - Denmark \\ jon83@kom.aau.dk
}

\author{
Frank H.P. Fitzek \\ Aalborg University \\ Dept. of Electronic Systems \\ Aalborg - Denmark \\ ff@es.aau.dk \\ Marcos Katz \\ VTT \\ Technical Research Centre of \\ Finland \\ Oulu, Finland \\ marcos.katz@vtt.fi
}

\begin{abstract}
Voice-over-IP (VoIP) has rapidly gained popularity in the last years, especially on smartphones platforms. In fact, WLAN chip-sets are becoming more common on smartphones, allowing users to use VoIP services from their mobile devices. On the other hand, the excessive energy consumption of the WLAN chip-set, limits the widespread use of VoIP on mobile phones. As most of the energy is spent when the WLAN is in idle mode waiting for a call, a possible solution is to use a secondary air interface, with lower energy consumption, as a signalling channel to wake up the WLAN radio. In this paper we describe two approaches to address this problem, namely the overlay and cooperative approaches.

We present measurements of energy consumption done on a commercial device to show that the presented approaches can reduce the energy consumption for VoIP calls on smartphones over WLAN.
\end{abstract}

\section{Categories and Subject Descriptors}

C.2.1 [Network Architecture and Design]: Wireless communication

\section{General Terms}

Energy saving, performance

\section{Keywords}

Mobile devices, cooperation, wireless networks, power measurements, wake up system, overlay networks

Mobimedia 2008 July 7-9, 2008, Oulu, Finland.

Copyright 2008 ICST ISBN 978-963-9799-25-7/08/07

DOI 10.4108/ICST.MOBIMEDIA2008. 4022

\section{INTRODUCTION}

In the last years, Voice-over-IP services have become very popular and seem to be very appealing if compared to traditional voice services. Applications such as Skype, Google Talk, MNS Messenger allow users to make free calls within the same community, offering also cheap fares for calling users outside the community. In addition, software like VoipStunt, VoipBuster, VoipDiscount offer the possibility to call landline phones in some countries, free of charge. Some of these software solutions have been ported to the smartphone platforms giving the users an alternative to the traditional call over the cellular network. Other software such as Fring enables the phone to have access to all these communities using the same client. Moreover smartphones are becoming more powerful and it is very common on the new models to have a Wi-Fi chip-set. This means that the user can use the VoIP services connecting the phone to a WLAN access point not only at home, but wherever there is a Wi-Fi hotspot. This will boost the VoIP services and it will benefit to the phones' manufacturers because the demand for devices with such capabilities will increase. One important aspect to take into consideration is the power consumption. Modern smartphones have several wireless interfaces, high definition cameras, GPS receivers which consume significant amounts of power. Using all of them at the same time causes a dramatic heating of the mobile phone, reducing drastically the operating time of the wireless device. The manufacturers use materials able to distribute the heating over the device in order to dissipate the heat as much as possible to protect the hot spots. However there is a threshold of temperature after which an active cooling system might be needed to guarantee that the temperature of the device does not exceed some practical limits. Figure 1 shows a temperature rise of 10 degree Celsius by changing the state of a Nokia N95 from "flying mode" (left) to transmitting data over WLAN (right).

Moreover the mobile phones are battery driven, which means that the energy resources are very limited. One solution would be to have batteries with more capacity, but unfortunately the evolution of the batteries does not follow 


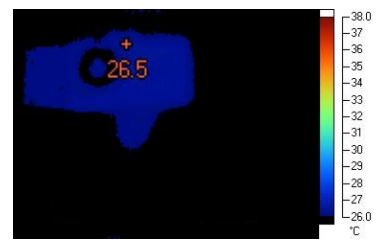

(a) N95 in offline mode. The maximum value of the temperature of the phone is $26.5^{\circ} \mathrm{C}$.

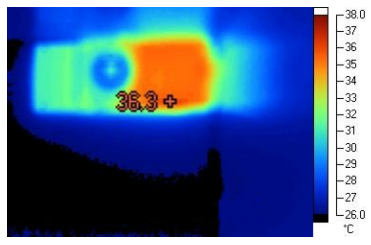

(b) N95 sending data with WLAN. The temperature can reach more than $36.3^{\circ} \mathrm{C}$.
Figure 1: Temperature distribution of a commercial wireless device (Nokia N95) in off-line mode and using WLAN

Moore's law. While the computational complexity is doubled every two years according to Moore, the battery capacity is doubling every decade. For the widespread adoption of VoIP on smartphones, a solution to extend the battery life is needed. When a smartphone is waiting for an incoming call using VoIP over $\mathrm{Wi}-\mathrm{Fi}$, the network interface on the phone has to be on all the time. Unfortunately the WLAN interface has an high energy consumption even when no data are transmitted. Therefore a huge amount of energy is spent on the idle state, just waiting for an incoming call. On the other hand, disconnecting from the network will not allow to detect incoming calls. In this paper we show the potential of using a wake-up system for WLAN systems when incoming VoIP call are expected. Section 2 gives an overview of the scenario. In Section 4 we show the benefit of using one of the proposed approach, namely overlay approach as a result of an analytical approach combined with the values obtained by the energy measurements on the Nokia N95 presented in Section 3.

\section{SCENARIO OVERVIEW}

In Figure 2 an overview of a VoIP activity is shown to understand the main potential of energy savings throughout this paper. We can distinguish several intervals having the same characteristics, namely calling frames. Within a calling frame there are two sessions:

Waiting session: typically there are long periods of inactivity where the phone is connected to the SIP server and waiting for a call.

Calling session: during this interval the phone receives the VoIP call. The duration of this interval highly depends on the user habits.

Regardless the duration of the two sessions, it is clear that the phone uses more power during the calling session. However, the waiting session can be very long meaning that the phone will spend more energy even though the power level is small. Measurements done in [6] show that having a WLAN connection open causes an exhaustive consumption of energy, even when no data is transmitted. Switching off the IEEE802.11 card in this case will not be an option as incoming calls cannot be received. In [2],[9], [1], authors have shown the benefit in terms of energy saving of using a low power air interface to wake up the higher one. This allows the high power air interface to be switched off for a longer period, and be woken up only when needed. We advocate two strategies for saving energy when using IEEE802.11 for

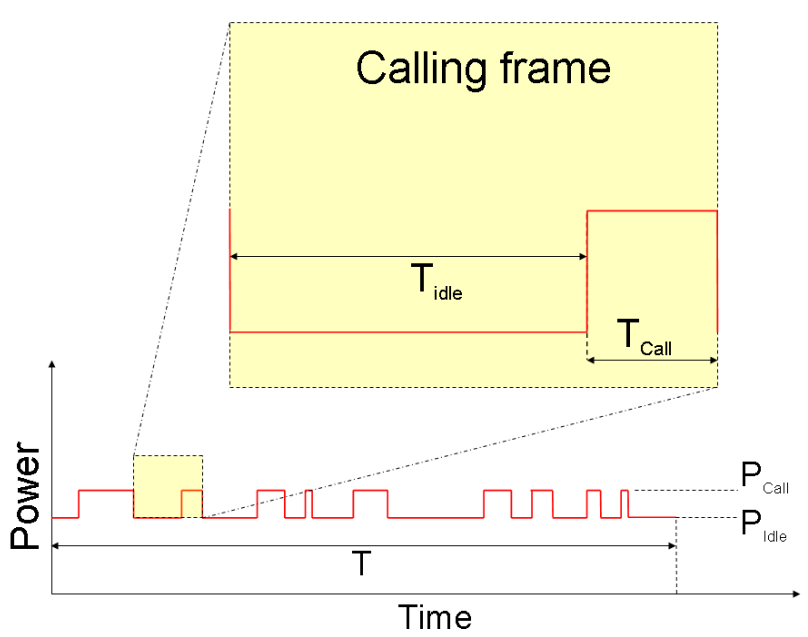

Figure 2: VoIP activity can be viewed as a collection of calling frames. Each of them is divided into waiting session and calling session.

VoIP applications: Overlay approach and Cooperative approach.

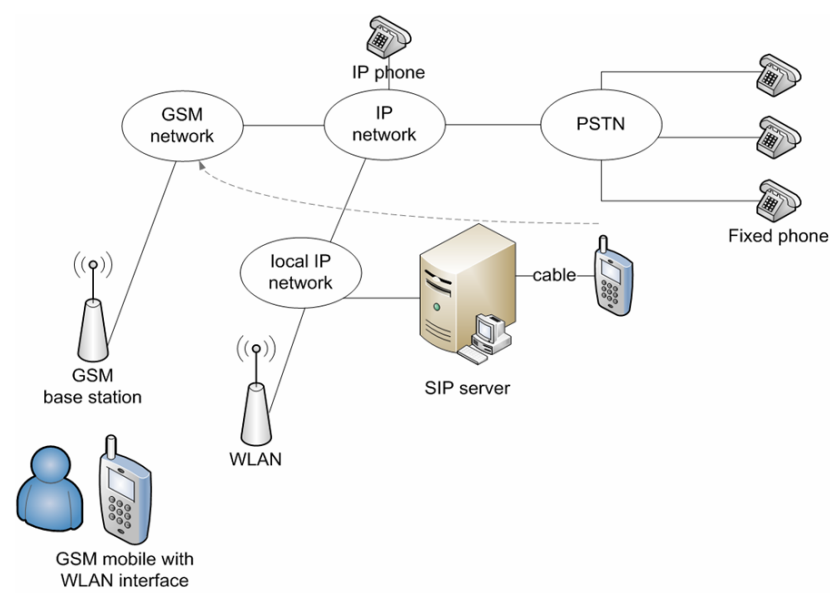

Figure 3: Overlay scenario for energy saving with wake up call.

\subsection{Overlay scenario}

Figure 3 shows a possible implementation of a system based on the Overlay approach. Let us suppose to have a call from a VoIP client (sender) to a mobile phone (receiver) routed through a SIP server. Let us also suppose that the server has a mapping of the GSM number and VoIP ID of the recipient device. The SIP server, before routing the call to the recipient mobile phone, will call its GSM number over the cellular network $(2 \mathrm{G} / 3 \mathrm{G})$. Upon receiving the call, the recipient mobile phone will identify the phone number of the server, understand that it is a wake-up call and suppress the incoming call by the server. From the mobile 
phone's perspective the incoming call by the server, identified by a certain number, is enough to understand that it should wake up. It will now switch on the IEEE802.11 card, connect to the access point and wait for the incoming call. In case of high degree of mobility the wireless device could also ping the SIP server to make his new connection details available. As soon as the recipient devices has logged in, the server can route the call. In this implementation, the calling session slightly differs from the traditional scenario where the recipient phone is continuously connected to the access point. As can be seen in Figure 4 the phone spends some extra energy every time it receives a call to set up the connection.

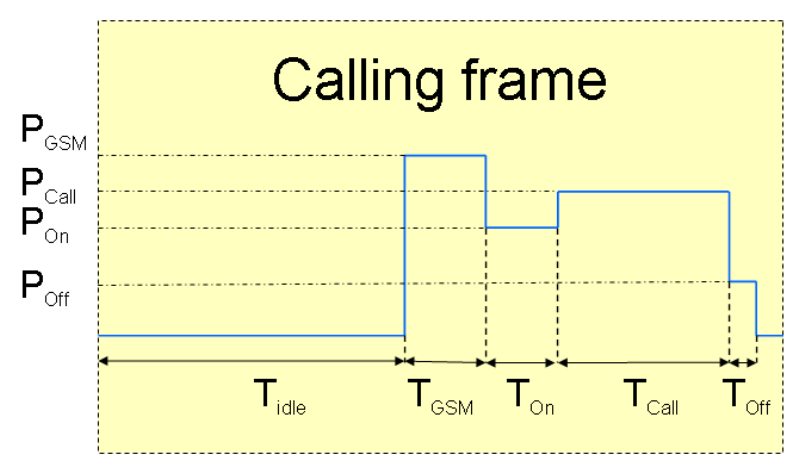

Figure 4: Calling frame for the Overlay approach.

The amount of energy spent during the calling session can be calculated as:

$E_{T o t}=P_{G S M} \cdot T_{G S M}+P_{O n} \cdot T_{O n}+P_{C a l l} \cdot T_{C a l l}+P_{O f f} \cdot T_{O f f}$,

where $P_{G S M}$ and $T_{G S M}$ are respectively the power spent and the time needed for receiving and suppress the incoming GSM wake up calls. $P_{O n}$ and $T_{O n}$ are respectively the power spent and the time needed for switching on the WLAN card and connecting to the access point. $P_{C a l l}$ and $T_{\text {Call }}$ are respectively the power spent and the time needed for making the VoIP call. Finally $P_{O f f}$ and $T_{O f f}$ are respectively the power spent and the time needed for disconnecting from the access point and switching off the WLAN card. As mentioned before, the duration and the energy spent for the VoIP call itself does not depend on the approach we use, therefore in order to compare the Overlay approach to the traditional one, we can take out the energy spent for the call from Equation 1. Equation 2 shows the formula for calculating the extra amount of energy spent per call with the Overlay approach.

$$
E_{\text {Setup }}=P_{G S M} \cdot T_{G S M}+P_{O n} \cdot T_{O n}+P_{O f f} \cdot T_{O f f}
$$

\subsection{Cooperative scenario}

A second approach for energy saving by wake-up calls is discussed here. This approach is only accessible for users that are able to cooperate among each other. Due to the space limitations we will not go into detail for cooperative wireless networks, but the interested reader is referred to [5]. For the ease of understanding, let us consider a group of users gathered in the same place within a short-range distance. Let us assume that all their phones are close to each other and that they are all waiting for an incoming VoIP call over WLAN. This could be for example a typical office scenario. To save energy some of the mobile phones in the group can switch off their WLAN, whereas one of them, referred as watch dog, can be connected to the access point. Whenever the access point has an incoming call for a device in the group, the watch dog will notify the recipient of the call to switch on the WLAN and to connect to the access point. Figure 5 shows a notification system based on RFID or UHF technology [4]. The system could be improved using coded messages to wake up dedicated devices.

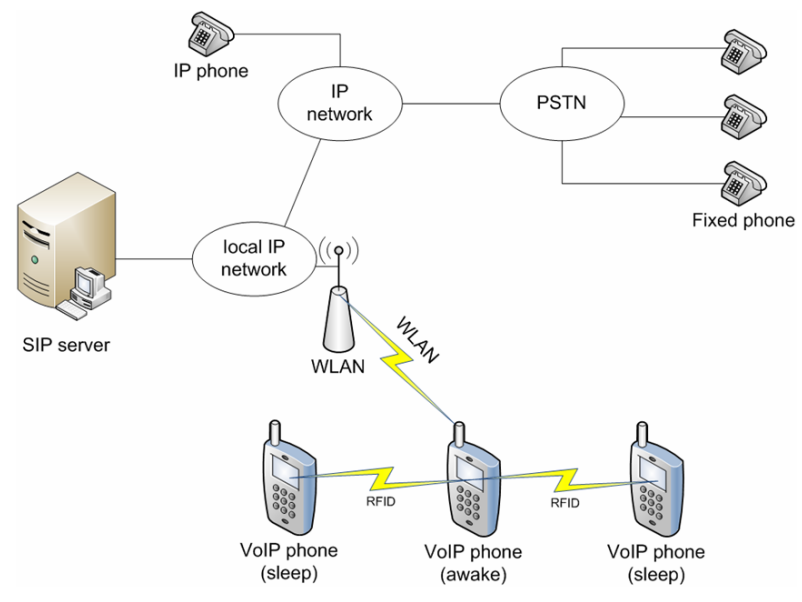

Figure 5: Notification system for a cooperative scenario. The watch dog upon receiving an incoming call for one of the mobile phones in the group, wakes them up using RFID or UHF technology.

The notification system can be done using different technologies as long as they are more energy efficient compared to WLAN. For example Bluetooth could be a choice for signalling as it consumes less power than WLAN [1]. Many smartphones, including the Nokia N95, have a built-in accelerometer which could be used for wake up. If the group of phone is lying on the same surface, on a table for example, the watch dog could vibrate and the phones will detect the vibration with the accelerometer and wake up. Another idea could be to use the light sensor embedded in some phones which can retrieve the light intensity. The watch dog can flash the phones with the camera flash to wake them up.

\section{MEASUREMENTS AND RESULTS}

In order to quantify the values of the single parameters in $(2)$, some measurements have been carried out. In this section the measurements setup and the results are presented. The smartphone used for the measurements is a Nokia N95 which is running Symbian OS as operating system. We used Python for S60 [8], [7] as programming language to develop the scripts for testing. We claim here that there is no significant penalty in terms of energy and performance by using the Python environment compared to standard Symbian/C++ compared to the energy levels we deal with throughout this paper. The choice of the mentioned 
commercial device is due to several reasons. First of all it is equipped with an IEEE802.11 card. Secondly it is able to run the in-built energy profiler [3] developed by Nokia. The energy profiler is an application on the mobile device that allows to make measurements without any additional hardware. It gives directly the values for power with a sample rate of $250 \mathrm{~ms}$. However, to check the correctness of the data carried out by the energy profiler on the phone, the complete setup includes the AGILENT 66319D used as multimeter as shown in Figure 6. It is connected to a $\mathrm{PC}$ which is running the Agilent 14565B device characterization software, a tool designed for evaluation of portable battery powered device current profiles.

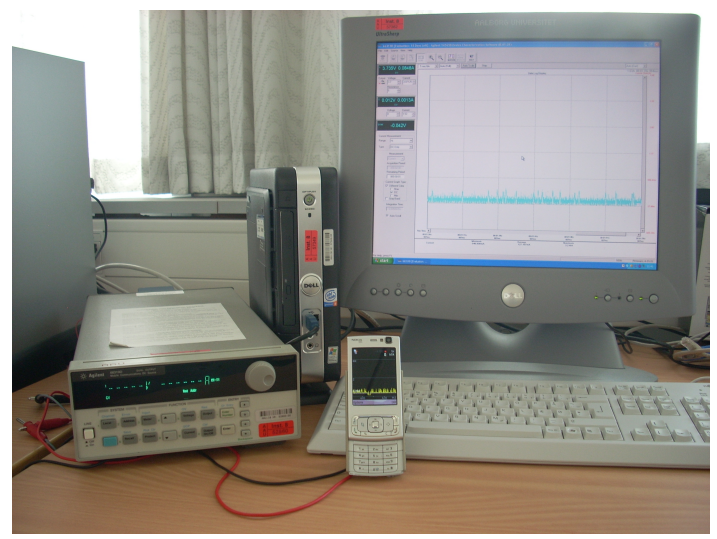

Figure 6: Setup for the measurements. The N95 connected to the AGILENT 66319D equipment.

We have compared results obtained with the energy profiler with the ones obtained with the Agilent 66319D. Figure 7 shows that the two plots match almost perfectly with each other proving that data given by the energy profiler are reliable.

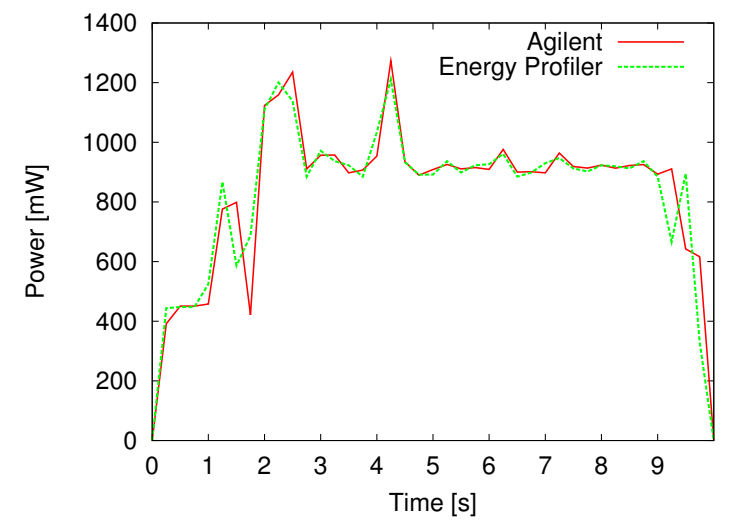

Figure 7: Comparison of data obtained with the energy profiler and the Agilent 66319D while measuring the connection of the phone to the WLAN access point.

We have tested every single action performed by the phone during the calling session of the Overlay approach separately. Each test has been running several times and figures in the following subsections show the plot of the mean value of these runs. Moreover the mean value of the power consumption and the time duration for each single action has been calculated. In all the measurements we do not take into account the energy spent for the backlight of the display and the ringtone in case of the incoming GSM call. Moreover during the measurements the phone was always connected to the GSM network to better emulate the real case and the other air interfaces, such as Bluetooth, were switched off. Figure 8 shows the plot of the power consumption over the time for receiving and suppressing an incoming GSM call. During this test the WLAN card was switched off. Figure 9 shows the plot of the power consumption over

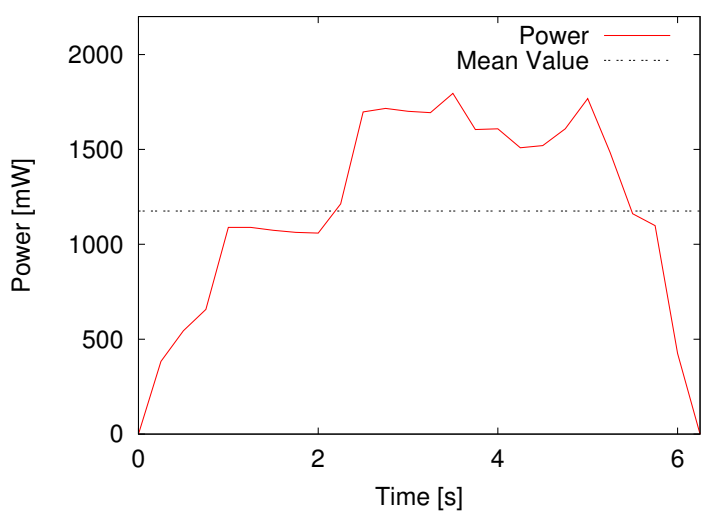

Figure 8: Power consumption for receiving and suppressing an incoming GSM call.

the time for connecting to the WLAN access point. The test consists of two steps: switching on the WLAN card and connecting to the access point. We have assumed that the phone connects to a predefined access point without searching. Figure 10 shows the plot of the power consumption over the time for disconnecting the phone from the access point. Figure 11 shows the plot of the power consumption over the time when the phone is connected to an access point but is not transmitting nor receiving data. This is the case of the waiting session with the traditional approach. Figure 12 shows the plot of the power consumption over the time when the phone is connected to the GSM network in idle mode. This is the case of the waiting session with the Overlay approach.

\section{DISCUSSION}

In order to compare the conventional approach with the overlay one, we need to calculate the energy spent by the two approaches. The energy for the conventional approach is given by Equation 3

$$
E_{\text {Trad }}=E_{W L A N, i}+N \cdot E_{\text {Call }},
$$

where $E_{W L A N, i}$ is the energy spent for being connected to a WLAN access point, $N$ is the number of calls during an interval of time $T$ and $E_{\text {Call }}$ is the energy spent during the 


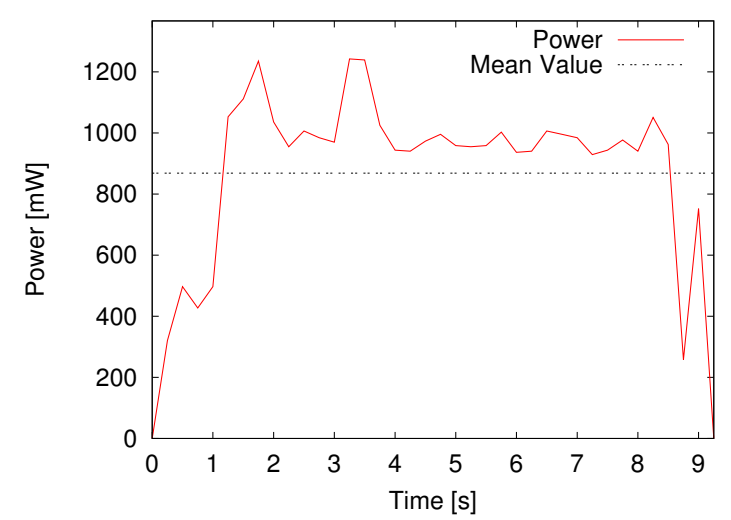

Figure 9: Power consumption for connecting to the WLAN.

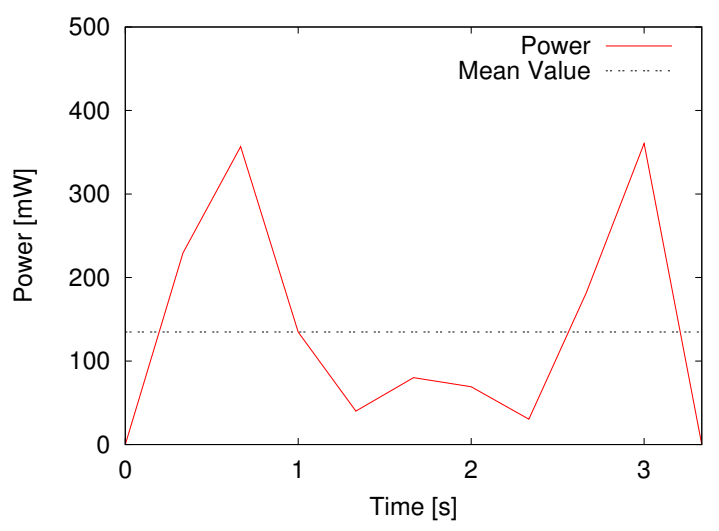

Figure 10: Power consumption for disconnecting from the WLAN.

VoIP call itself. Equation 4 shows how to calculate the energy spent in the Overlay approach.

$$
E_{\text {Over }}=E_{G S M, i}+N \cdot E_{\text {Call }}+N \cdot E_{\text {Setup }},
$$

where $E_{G S M, i}$ is the energy spent being coneected to the GSM network and in idle mode and $E_{\text {Setup }}$ is the energy in Equation 2. Equation 5 shows the energy gain as a difference of $E_{\text {Trad }}$ minus $E_{\text {Over }}$.

$$
E_{\text {Gain }}=E_{W L A N, i}-E_{G S M, i}-N\left(E_{\text {Setup }}\right),
$$

where

$$
E_{W L A N, i}=\left(T-N \cdot T_{C a l l}\right) \cdot P_{W L A N, i}
$$

and

$$
E_{G S M, i}=\left(T-N \cdot T_{\text {Call }}-N \cdot T_{\text {Setup }}\right) \cdot P_{G S M, i} .
$$

If we define a gain factor $\mathrm{G}$ as:

$$
G=P_{W L A N, i}-P_{G S M, i}=0.0832 W
$$

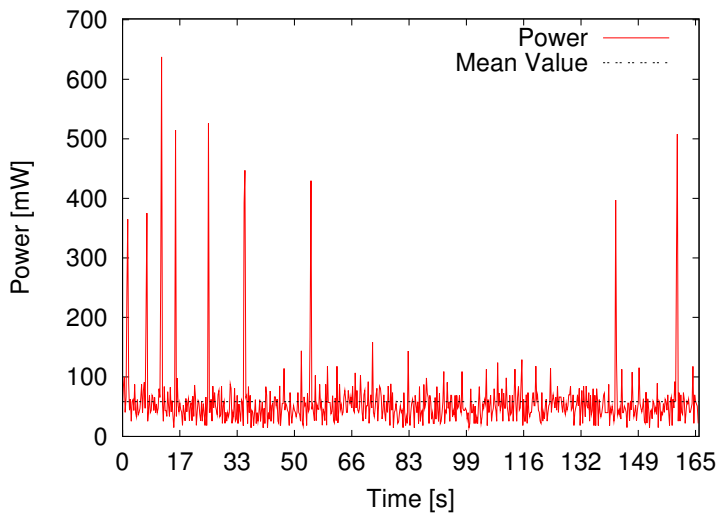

Figure 11: Power consumption when connected to the WLAN and being in idle mode.

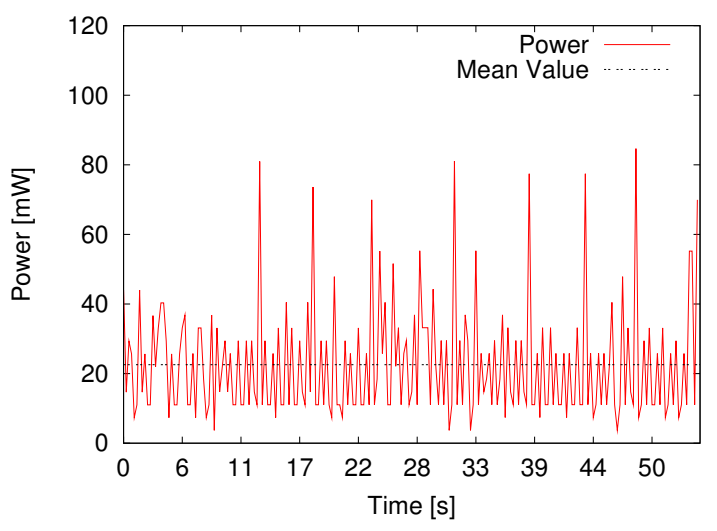

Figure 12: Power consumption when being connected to the GSM network with no activity.

by replacing the expressions of Equations 2, 6 and 7 in Equation 5, we obtain Equation 9.

$E_{\text {Gain }}=T \cdot G-N\left(T_{\text {Call }}\right) \cdot G+N\left(T_{\text {Setup }} \cdot P_{G S M, i}-E_{\text {Setup }}\right)$

Plugging in the values for $T_{\text {Setup }}, P_{G S M, i}, E_{\text {Setup }}$ of Table 1 in Equation 9 we obtain the cost function of the energy gain in Equation 10:

$$
E_{\text {Gain }}=G \cdot T-N\left(T_{\text {Call }} \cdot G+16\right),
$$

where $T$ is the duration of the all Voip activity as in Figure 2, $N$ is the number of calls received within the time $T$ and $T_{\text {Call }}$ is the mean value of the duration of the calls. Plugging in Equation 10 the value of $G$ from Equation 8, the gain is greater then zero when:

$$
N<\frac{T}{T_{\text {Call }}+418}
$$


Table 1: Power consumption, duration and energy of an N95 for different scenarios

\begin{tabular}{|l|r|r|r|}
\hline Scenario & Power & Time & Energy \\
\hline Incoming GSM call & $1175.4 \mathrm{~mW}$ & $6.5 \mathrm{~s}$ & $7.6 \mathrm{~J}$ \\
\hline Connecting to WLAN & $868.5 \mathrm{~mW}$ & $9.5 \mathrm{~s}$ & $8.2 \mathrm{~J}$ \\
\hline Disconnecting from WLAN & $134.8 \mathrm{~mW}$ & $2.8 \mathrm{~s}$ & $0.4 \mathrm{~J}$ \\
\hline Connected to WLAN - idle & $58.4 \mathrm{~mW}$ & & \\
\hline GSM - idle & $20.2 \mathrm{~mW}$ & & \\
\hline
\end{tabular}

Table 2: Energy gain examples for different values of $\mathrm{T}$, $T_{\text {Call }}$ and $\mathbf{N}$.

\begin{tabular}{|l|r|r|r|r|}
\hline User & $T_{\text {Call }}$ & $\mathrm{T}$ & $\mathrm{N}$ & $E_{\text {Gain }}$ \\
\hline User 1 & $15 \mathrm{~min}$ & $8 \mathrm{~h}$ & 25 & $124 \mathrm{~J}$ \\
\hline User 2 & $10 \mathrm{~min}$ & $8 \mathrm{~h}$ & 10 & $1737 \mathrm{~J}$ \\
\hline User 3 & $7 \mathrm{~min}$ & $8 \mathrm{~h}$ & 5 & $2141 \mathrm{~J}$ \\
\hline
\end{tabular}

In Figure 13 the cost factor $\mathrm{C}=\frac{T}{T_{\text {Call }}+418}$ is plotted for different values of $T$. Fixed $T$, for a given $T_{\text {Call }}, E_{\text {Gain }}$ is positive

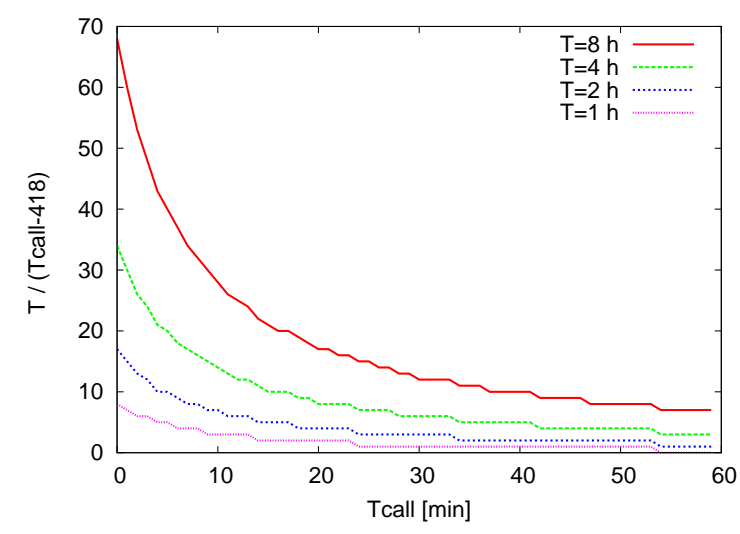

Figure 13: Cost factor $C$ plotted for different values of $\mathbf{T}$.

if $N$ is smaller than the cost factor $C$. Let us suppose to have three users using VoIP services on the mobile devices. In Table 2 some possible combinations of values for $T, T_{\text {Call }}$ and $N$ are shown. In all the three cases the energy gain is greater than zero even for the User 1 which has a high mean duration of the calls and receives 25 calls within 8 hours.

\section{CONCLUSIONS}

In this paper we presented a new approach to reduce the excessive energy consumption of the WLAN interface of a smartphone in a VoIP scenario. The use of a secondary air interface to wake up the WLAN has good potential for saving energy. Two different ways of implementing a wake up system have been presented, namely overlay and cooperative approach. Analytical results show the benefit of the overlay approach. This has been supported by measurements of energy consumption on an Nokia N95 while performing different tasks. Finally three different scenarios have been analyzed showing that the wake up system can reduce significantly the energy consumption of a mobile phone in a VoIP scenario when compared to the conventional approach. It has been shown that the overall gain of the proposed approaches depends heavily on the users' call activity. Nevertheless for a broad range of user call activities a gain by the different approaches has been presented.

\section{ACKNOWLEDGMENTS}

Authors would like to thank Nokia for providing technical support as well as mobile phones to carry out the measurement campaign. Special thanks to Mika Kuulusa, Gerard Bosch, Harri Pennanen, Nina Tammelin, and Per Moeller from Nokia. This work was partially financed by the X3MP project granted by Danish Ministry of Science, Technology and Innovation.

\section{REFERENCES}

[1] Y. Agarwal, S. C., and G. R. Dynamic power management using on demand paging for networked embedded systems. In In Proc. of Asia-South Pacific Design Automation Conference (ASPDAC), 2005.

[2] Y. Agarwal, R. Chandra, A. Wolman, P. Bahl, K. Chin, and R. Gupta. Wireless wakeups revisited: energy management for voip over wi-fi smartphones. Proceedings of the 5th international conference on Mobile systems, applications and services, 2007.

[3] G. Bosh and M. Kuulusa. Mobile Phone Programming and its Application to Wireless Networking, chapter Optimizing mobile software with buit-in power profiling.

[4] P. Calton, W. Bamford, F. Chehimi, P. Gilberstson, and O. Rashid. Mobile Phone Programming and its Application to Wireless Networking, chapter Using In-built RFID/NFC, Cameras, and 3D Accelerometers as Mobile Phone Sensors. Springer, 2007.

[5] F. Fitzek and M. Katz, editors. Cooperation in Wireless Networks: Principles and Applications - Real Egoistic Behavior is to Cooperate! ISBN 1-4020-4710-X. Springer, April 2006.

[6] M. Petersen, G. Perrucci, and F. Fitzek. Energy and Link Measurements for Mobile Phones using IEEE $802.11 \mathrm{~b} / \mathrm{g}$. In The 4th International Workshop on Wireless Network Measurements (WiNMEE 2008) - in conjunction with WiOpt 2008, Berlin, Germany, Mar. 2008.

[7] J. Scheible. Mobile Phone Programming and its Application to Wireless Networking, chapter Python for symbian phones. Springer, 2007.

[8] J. Scheible and V. Tuulos. Mobile Python: Rapid Prototyping of Applications on the Mobile Platform. Wiley, ISBN: 978-0-470-51505-1, 2007.

[9] E. Shih, P. Bahl, and M. J. Sinclair. Wake on wireless: An event driven energy saving strategy for battery operated devices. In Proceedings of the Eighth Annual ACM Conference on Mobile Computing and Networking, 2002. 